Original Article

\title{
Knowledge, Attitude and Practice (KAP) Of Spread of HIV Infection through Needle Stick Injury among Dental Paramedics
}

\author{
Nidarsh Hegde', Freddy Kersi M istry ${ }^{2}$, Soumi Samuel ${ }^{3} \&$ S.M. Sharma ${ }^{4}$ \\ ${ }^{1}$ Professor, ${ }^{2}$ Post Graduate, ${ }^{3}$ Reader, ${ }^{4}$ Professor \& HOD, Department of Oral \& M axillofacial Surgery, \\ A.B. Shetty M emorial Institute of Dental Sciences, Nitte University, M angalore, Karnataka, India. \\ Correspondence \\ Freddy Kersi Mistry \\ Post Graduate, Department of Oral \& Maxillofacial Surgery, \\ A.B. Shetty M emorial Institute of Dental Sciences, Nitte University, Mangalore 575 018, Karnataka, India \\ Mobile : +91 9900409342 E-mail : drfreddymistry@gmail.com
}

\begin{abstract}
Aim: Study was conducted to know the knowledge of the paramedics working at Dental Institute, regarding spread of HIV infection through needle stick injury. Their current practice for the management of needle stick injury and their attitude for the same.

M aterials and M ethod : 100 paramedical staff working in a Dental Institute were selected randomly to participate in the study. Each one of the participant were requested to fill a structured survey form prepared by the surveyor reflecting their knowledge, practice and attitude of spread of HIV infection through needle stickinjury.

Results : The statistical analysis revealed the $92 \%$ of the paramedical staff knows what HIV infection is and that it can be spread through needle stick injury irrespective of their academic background. $42 \%$ of the paramedics have experienced needle stick injury, among which $95 \%$ have experienced $1-2$ times a year. $61 \%$ of the needle stick injury have been reported by the paramedics with majority of them reporting to the head of the department followed by colleagues. According to the survey $95 \%$ of the paramedics followed the correct post injury protocol even though only $90 \%$ of them knew how to manage it correctly. $88 \%$ of the paramedics have been educated regarding the management of needle stick injury through the programmethey have attended conducted by their respective institution.

Conclusion : This survey revealed that know ledge of paramedics about the risk association of spread of HIV infection with needle-stick injuries and use of preventive measures was adequate. A standing order procedure (SOP) which if formulated regarding needle-stick injuries should be further encouraged by the head of the respective departments especially to those who have newly joined the institution to obtain still better safety measures.
\end{abstract}

Keywords : Dental Paramedics, Human Immunodeficiency Virus(HIV), Needle Stick Injury (NIS).

\section{Introduction}

Dental surgeons are trained to follow a strict infection control protocol, as it has been inculcated in a trained professional through their academic curriculum. But in a dental hospital set up it's not just a dental surgeon who runs the entire show, theirs a major role even played by the dental paramedics.

Dental paramedics are equally exposed to needle stick

Access this article online

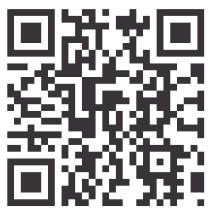

injury as much as a dental surgeons. In order to prevent the spread of HIV infection through the mode of needle stick injury among paramedics it is important to know how much knowledge do they have regarding this mode of spread, how strictly they follow the departmental protocol and also to know their attitude for the same.

Studies conducted in various other countries showed that knowledge of health care workers about the risk asso ciated with needle-stick injuries and use of preventive measures was inadequate. A standing order procedure (SOP) should be formulated regarding needle-stick injuries in all the health institutions. ${ }^{1-5}$

As most of the studies regarding spread of HIV through needle stick injury are done on the medical paramedics we found a need to know the knowledge, attitude and practice of the dental paramedics as they are equally prone to such injury in day to day practice. 


\section{Materials and Methods}

\section{Sample selection}

A list of paramedical staff working at A. B. Shetty Memorial Institute of Dental Sciences, Mangalore from 2013-2014 was obtained from the institution office. A total of 100 paramedical staff were selected randomly who were doing different nature of workin different departments.

\section{Methodology}

A structured form of 14 questions was constructed by the surveyor and was distributed among the samples to know their knowledge, attitude and practice regarding the spread of HIV infection through needle stick injury.

The form was prepared in both the languages i.e. Universal language English and Local regional language i.e. Kanadda.

The samples were told to fill the form in front of the surveyor to prevent any influential bias.

\section{Results}

Demographic Characteristics of the paramedical staff working at institute

Table 1 : Demographic Characteristics

\begin{tabular}{|l|c|}
\hline Demographic Characteristics & Number \\
\hline 1.) Sex a.) M ale & 22 \\
b.) Female & 78 \\
\hline 2.) Age a.) $18-30$ & 3 \\
b.) $31-40$ & 44 \\
c.) $41-50$ & 46 \\
d.) $51-60$ & 7 \\
\hline 3.) Duration of their association to the institute & \\
a.) $0-5$ & 12 \\
b.) 6-10 & 18 \\
c.) 11-15 & 06 \\
d.) 16-20 & 18 \\
e.) $21-25$ & 34 \\
f.) 26-30 & 12 \\
\hline a.) Oral M edicine and Radiology & 10 \\
b.) Prosthodontics & 20 \\
c.) Conservative Dentistry and Endodontic & 20 \\
d.) Periodontics & 10 \\
e.) Orthodontics & 10 \\
f.) Pedodontics & 10 \\
g.) Oral and M axillofacial Surgery & 10 \\
h.) Oral Pathology & 05 \\
i.) Preventive and Public Health Dentistry & 05 \\
\hline
\end{tabular}

Fig. 1: Distribution of Nature of Work Among the total sample size of 100; 37 worked in sterilization area, 24 were sweepers, 14 were receptionist, 13 were laboratory technicians, 9 were chair side attender, 3 were dental nurse. (Fig. 1)

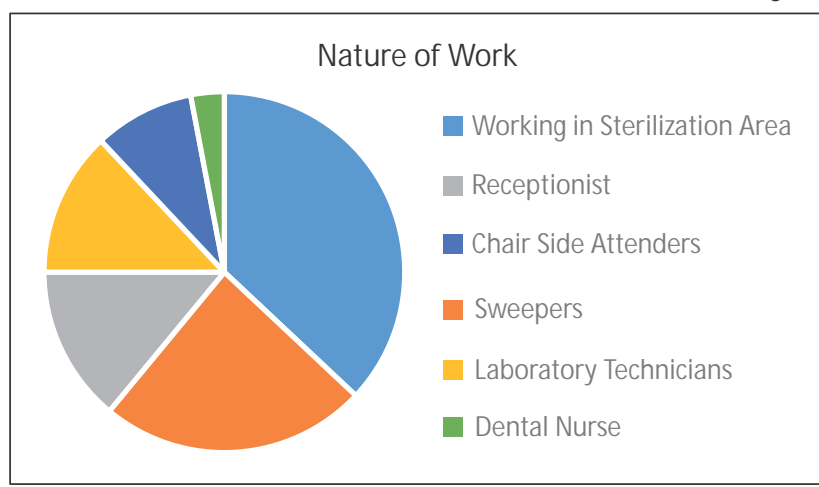

Fig. 2: Distribution of Academic Qualification

When their educational qualification was surveyed it was observed that majority i.e. $58 \%$ have not completed their 10th std., $14 \%$ had finished their education till 10th std. and 18\% till 12th std., $5 \%$ had finished their graduation and $5 \%$ had got a degree in Dental Technician course. (Fig. 2)

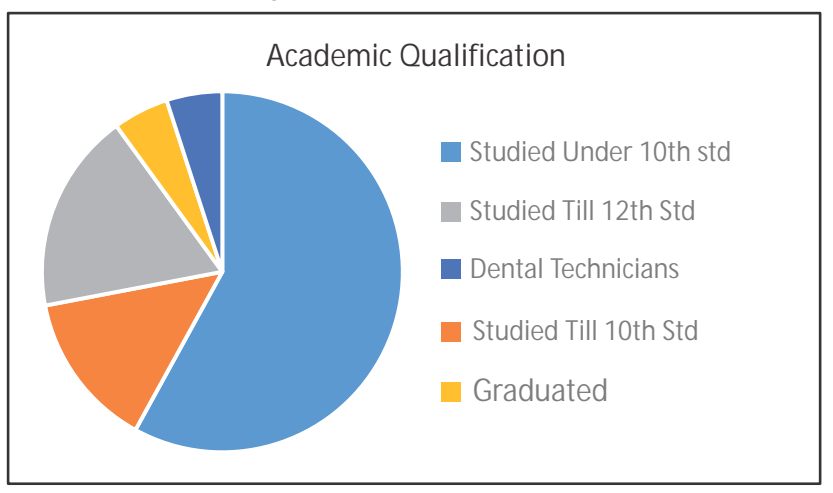

Table 2: Level of knowledge and preventive measures taken by paramedical staff regarding needle stickinjury

\section{Occupational Hazards and Preventive Measures $\quad$ Number}

1.) Do you know what HIV infection is?
a.) Yes
b.) No
92
08

2.) Have you ever experienced Needle Stick Injury?
a.) Yes
b.) No 58

3.) What is the frequency of Needle Stick Injury per year?
a.) $1-2$
b.) $3-4$
$40 / 42$
c.) $5-6$
$02 / 42$
00

4.) Have you reported the incident of Needle Stick Injury

a.) Yes

b.) No

$26 / 42$

$16 / 42$

5.) Do you know how to manage Needle Stick Injury?
a.) Yes
b.) No 
$92 \%$ of the paramedical staff knows what HIV infection is and that it can be spread through needle stick injury irrespective of their academic background. $42 \%$ of the paramedics have experienced needle stick injury, among which $95 \%$ have experienced $1-2$ times a year. $61 \%$ of the needle stick injury have been reported by the paramedics with majority of them reporting to the head of the department followed by colleagues. According to the survey $95 \%$ of the paramedics followed the correct post injury protocol even though only $90 \%$ of them knew how to manage it correctly. $88 \%$ of the paramedics have been educated regarding the management of needle stick injury through the programme they have attended conducted by their respective institution.

\section{Discussion}

In this study out of 100 paramedics who participated $92 \%$ were aware that HIV infection can be transmitted through needle stick injury. A data combined from more than 20 prospective studies worldwide of health care workers exposed to HIV-infected blood through percutaneous injury revealed an average transmission rate of $0.3 \%$ per injury. ${ }^{6}$ One study reported that transmission occurred only from hollow-bore needles as compared with other sharp objects.

Out of 42 paramedics experiencing needle stick injury, $61 \%$ of them have reported. Data from EPINet system suggest that at an average hospital, workers incur approximately 30

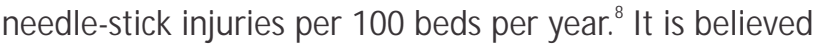

\section{References}

1. Gurubacharya DL, Mathura KC, Karki DB. Knowledge, attitude and practices among health care workers on needle-stick injuries. Kathmandu Univ M edJ (KUMJ). 2003 Apr-Jun; 1(2):91-4.

2. Khurram Siddique, Shirin Mirza, Syeda Fizza Tauqir, Idress Anwar, Asif Zafar M alik. Knowledge, attitude and practice regarding needle stick injuries amongst healthcare providers. Pakistan Journal of Surgery. 2008 Vol 24 (4): 243-48.

3. Gumodoka B, Favot I, Berege ZA, Dolmans WM. Occupational exposure to the risk of HIV infection among health care workers in Mwanza Region, United Republic of Tanzania. Bull World Health Organ. 1997; 75(2):133-40.

4. Askarian M, Malekmakan L. The prevalence of needle stick injuries in medical, dental, nursing and midwifery students at the university teaching hospitals of Shiraz, Iran. Indian J Med Sci. 2006 Jun; 60(6):227-32.

5. Muralidhar S, Singh PK, Jain RK, Malhotra M, Bala M. Needle stick injuries among health care workers in a tertiary care hospital of India. Indian J Med Res. 2010 M ar; 131: 405-10. that only one out of three needle-stick injuries are reported in the US, while these injuries virtually go undocumented in many developing countries. ${ }^{9}$

The circumstances leading to needle-stick injury depend partly on the type and design of the device and certain work practices. In this study $84 \%$ of the paramedics wear double gloves when in contact with potential sharp objects, while in other studies they used double glove as precaution only in $23 \%$ of cases. ${ }^{1}$

In 1985, in order to increase awareness among health care workers of the dangers of sharp injuries and other types of disease transmission, the Centre for Disease Control (CDC) and the Occupational Safety and Health Administration (OSHA) in the United States introduced the "Universal Precaution Guidelines", which have been the worldwide standard in both hospital and community care settings. ${ }^{10}$ In our study we found that $90 \%$ of the participants knew how to manage needle stick injury, which is statistically high compared to various other studies. ${ }^{1,2,3,4}$

\section{Conclusion}

This survey revealed that knowledge of paramedics about the risk association of spread of HIV infection with needlestick injuries and use of preventive measures was adequate. A standing order procedure (SOP) which if formulated regarding needle-stick injuries should be further encouraged by the head of the respective departments especially to those who have newly joined the institution to obtain still better safety measures.

6. Gerberding JL. Incidence and prevalence of human immunodeficiency virus, hepatitis $B$ virus, hepatitis $C$ virus and cytomegalovirus virus among health care personnel at a risk of blood exposure: a final report from a longitudinal study. Infect Dis 1994; 170:1410-7.

7. Puro V, Petrosilla N, Ippolito G. Italian Study Group on Occupation Risk of HIV and other Blood-borne Infections. Risks of hepatitis $C$ seroconversion after occupational exposure in health care workers. Am J Infect Control 1995; 23: 273-7.

8. EPINet. Exposure Prevention Information Network data reports. University of Virginia: International Health Care Worker Safety Center.

9. Roy E, Robillard P. Under-reporting of accidental exposures to blood and other body fluids in health care setting: an alarming situation. Adv Exposure Prev 1995; 14: 11-3.

10. Centers for Disease Control. Perspective in disease prevention and health promotion update. Universal precautions for prevention of transmission of HIV, HBV and other blood-borne pathogens in health care settings. M M WR 1988; 37:24. 